\section{ROTEM for Diagnosing Hy- perfibrinolysis in Patients on the Liver Transplant Waiting} List

\author{
Scott G Allen ${ }^{1 *}$, Charles E Galaviz ${ }^{1}$, Nathan L Pace ${ }^{1}$, Kyle J \\ Sherwinz ${ }^{1}$ and Robin D Kim ${ }^{2}$
}

'Department of Anesthesiology, University of Utah, Salt Lake City, USA

${ }^{2}$ Department of Surgery, University of Utah, Salt Lake City, USA

\begin{abstract}
Background: Hyperfibrinolysis has been reported in $23-31 \%$ of patients with cirrhosis. This leads to excess bleeding and transfusion during liver transplantation. Rotational Thromboelastometry has recently become available in the United States and may offer quick diagnosis of hyperfibrinolysis. We aimed to assess the utility of the EXTEM and APTEM channels in diagnosing fibrinolysis in patients during the pre-transplant period to allow practitioners to decide whether to use these modalities to diagnose and treat with antifibrinolytics.
\end{abstract}

Methods: Blood samples were drawn from patients on the liver transplant waiting list, before surgery. APTEM and EXTEM tests were performed. Samples were run for 60 minutes. Statistical analysis included descriptive statistics and exact binomial test.

Results: Fifty-four patients completed the study. Median EXTEM maximum lysis was 5.0 (IQR 2.25-7.0). EXTEM ML > 15\% was found in $1 / 54$ patients $(1.8 \%)$. Using the exact binomial test for EXTEM ML>APTEM ML, $27 / 54(50 \%, 95 \% \mathrm{Cl} 0.36-0.63, p=1)$ met criteria for hyperfibrinolysis.

Conclusion: EXTEM maximum lysis, and EXTEM combined with

*Corresponding author: Scott G Allen, Department of Anesthesiology, University of Utah, Salt Lake City, USA, Tel: +1 8012054115; E-mail: Scott.allen@hsc. utah.edu

Citation: Allen SG, Galaviz CE, Pace NL, Sherwinz KJ, Kim RD (2017) ROTEM for Diagnosing Hyperfibrinolysis in Patients on the Liver Transplant Waiting List. J Anesth Clin Care 4: 023.

Received: September 25, 2017; Accepted: December 01, 2017; Published: December 18, 2017
APTEM give widely variable incidences of hyperfibrinolysis. These tests should not be used alone when diagnosing and treating hyperfibrinolysis. Further studies are needed to validate ROTEM when diagnosing hyperfibrinolysis in liver transplant recipients.

Keywords: Antifibrinolytics; Hyperfibrinolysis; Liver transplant; Thromboelastometry

\section{Introduction}

Hyperfibrinolysis is an ill-characterized coagulation disorder that can complicate liver transplantation, when bleeding and transfusion requirements can result in increased morbidity and mortality [1]. The coagulopathy associated with liver failure can either be one of hypocoagulation-related to decreased production of clotting factors or hypercoagulation due to decreased production of Proteins $\mathrm{C}$ and $\mathrm{S}$. Hyperfibrinolysis is prevalent in liver failure due to several factors. First, the liver does not clear tissue plasminogen activator and levels of this fibrinolytic enzyme increase. Second, plasminogen activator inhibitor (which binds to and inhibits tPA) is not synthesized by the failing liver [2]. Previous studies have shown that hyperfibrinolysis is present in $23-31 \%$ of cirrhotics [3].

The diagnosis of hyperfibrinolysis is difficult. Traditional methods, including the euglobulin lysis time and tPA levels are time consuming and costly, making their utility in clinical practice limited. Viscoelastic tests such as thromboelastometry and thromboelastography have been used more recently and have shown to be accurate tests for hyperfibrinolysis [4,5]. Still, hyperfibrinolysis remains an ill-defined phenomenon in the liver transplant population.

ROTEM (TEM Systems, Durham, NC, USA) has quickly gained acceptance in the United States since 2011, when it obtained Food and Drug Administration approval. It has been in use since 2003 in Europe. It allows testing of coagulation at the bedside. To perform the test, patient blood is drawn into a citrated tube. Whole blood is then placed into the ROTEM cup. A pin is rotated in the blood as it clots. As the clot forms, the pin slows down and a computer algorithm gives a graphical representation of the strength of the clot [6]. The EXTEM channel adds recombinant tissue factor to the sample, instigating the extrinsic clotting cascade. APTEM is performed by adding tranexamic acid (historically aprotinin) to the EXTEM sample and is used as an in vitro marker of resolution of fibrinolysis with antifibrinolytic therapy. Other tests can be performed by adding different reagents. The FIBTEM tests adds cytochalasin D to the EXTEM test, which inactivates platelets and gives a better representation of fibrinogen contribution to the clot $[7,8]$. Some contraction of the clot is expected, but in patients with hyperfibrinolysis, the clot strength diminishes rapidly.

Because of the high rate of hyperfibrinolysis, many centers use antifibrinolytic therapy during liver transplantation. This has shown to be safe, though the optimum dose and timing of therapy is unknown [9]. Thrombosis remains a dreaded complication so antifibrinolytics are used judiciously and are not standard of care in all centers. Our study sought to further characterize the incidence of hyperfibrinolysis 
in the pre-transplant period to further aid clinicians in their management decisions.

\section{Materials and Methods}

After obtaining institutional review board approval at the University of Utah, all patients on the liver transplant waiting list were identified. At any given time, there are approximately 90 patients on the University of Utah liver transplant waiting list. Patients were recruited between September 2015 and July 2016. All patients on the list were eligible to enroll in the study. Due to the large geographic area which the University of Utah covers, many patients live several hours from Salt Lake City and they did not make frequent visits to the hospital. The sample size of the participants was determined to be a convenient sample of all eligible patients, as this was an observational study.

Patients were captured at three times points: at the time of liver transplantation, during hospitalization, or immediately before transplantation. Patients were sampled only once. Sampling could occur at any time point from the time of initial listing up until the time of liver transplantation. All samples were drawn before any surgical intervention or coagulopathy correction in the operating room. After obtaining informed consent from the patient, a ROTEM sample was drawn. Blood was collected by venipuncture or line draws into citrated collection tubes. Fifty-eight patients were included in the study and had samples drawn. Four samples were not included in the analysis because the ROTEM was stopped before 60 minutes. This occurred due to operator error or because the ROTEM machine was needed for a critically ill patient.

Due to limited funding, and the manufacturer's recommendation that EXTEM be used to diagnose hyperfibrinolysis, only EXTEM and APTEM channels were performed. The blood samples were run within 1 hour of drawing the blood. Samples were only included in the study if they were run for 60 minutes, which would allow an adequate time for hyperfibrinolysis to manifest. Hyperfibrinolysis was defined as a maximum lysis on EXTEM of $>15 \%$ or APTEM maximum lysis greater than EXTEM maximum lysis. Maximum lysis is the decrease in clot strength, starting from the Maximum Clot Firmness. The definition of hyperfibrinolysis was based on manufacturer recommendations and available evidence at the time of study design. MELD score was used to stratify liver disease for two reasons: (1) this is the scoring system most commonly used to list patients for transplant, and it is based off of objective laboratory measurements. Cancer exception points were not calculated into our MELD scores for purposes of this study. The MELD presented the score of the patient, based on laboratory results, on the day of ROTEM testing, and is not necessarily the highest MELD reported throughout the patient's disease course.

\section{Statistical Analysis}

Simple descriptive statistics included means and standard deviations and medians with Interquartile Range (IQR). Statistical models, including exact binomial test and McNemar's test, were estimated and created in the R environment version 3.2.4 (R Core Team (2016)). R: A language and environment for statistical computing. R Foundation for Statistical Computing, Vienna, Austria. URL https://www.R-project.org/). Plots and correlation coefficients were created using Microsoft Excel version 15.28 (Redmond, Washington). Proportions are reported as decimal fractions with $95 \%$ CIs.

\section{Results}

Patient demographics are shown in table 1. Our center is in the American Intermountain West and the majority of patients were Caucasian. The major causes of liver failure were due to hepatitis C (27.7\%), alcoholic liver disease (25.9\%) and non-alcoholic steatohepatitis $(24.1 \%)$ (Table 2). Eleven patients (20.3\%) included in analysis had hepatocellular carcinoma complicating the underlying liver disease. Median EXTEM maximum lysis was 5.0 (IQR 2.25-7.0). By the definition of hyperfibrinolysis as EXTEM maximum lysis being greater than $15 \%$ (Table 3$), 1$ of 54 patients $(0.018 ; 95 \%$ CI [0.0005, 0.0989]) demonstrated hyperfibrinolysis. By the definition of hyperfibrinolysis as APTEM maximum lysis being greater than EXTEM maximum lysis, 27 of 54 patients $(0.5$; 95\% CI [0.36-0.63]) demonstrated hyperfibrinolysis. Another test of hyperfibrinolysis, APTEM Maximum Clot Firmness (MCF) MCF $>$ EXTEM. MCF was analyzed using the exact binomial test. In this test $24 / 54$ patients met the definition of enhanced fibrinolysis, but the results were not statistically significant $(\mathrm{p}=0.4966)$.

Comparison of the patients who exhibited hyperfibrinolysis under both definitions is best shown graphically in table 4 . There was wide discordance between the two definitions. Only one patient met the definition of hyperfibrinolysis in both.

As hyperfibrinolysis has been associated with worsening liver function, we assessed the correlation of EXTEM ML and MELD scores. There was poor correlation between EXTEM ML and MELD $\left(\mathrm{R}^{2}=0.095\right)$, with a trend toward less lysis as the liver failure progressed (Figure 1).

\begin{tabular}{|c|c|}
\hline Demographics & $\mathbf{N}=\mathbf{5 4}$ \\
\hline Age [years] & $57.5(28-72)^{*}$ \\
\hline Female & 28 \\
\hline Male & 26 \\
\hline Body mass index & $28.4(15.8-44.1)^{*}$ \\
\hline Meld & $18.0(6.0-44)^{*}$ \\
\hline INR & $1.5(0.9-3.9)^{*}$ \\
\hline Antiplatelet therapy & $2(3.7 \%)$ \\
\hline Platelets [K/Ul] & $99.0(22.0-131.5)^{*}$ \\
\hline Tips & $3(5.5 \%)$ \\
\hline White & 49 \\
\hline Native American & 3 \\
\hline Asian & 1 \\
\hline Hispanic & 15 \\
\hline
\end{tabular}

Table 1: Demographics of participants.

MELD: Model of End-Stage Liver Disease; INR: International Normalized Ratio; TIPS: Transjugular Intrahepatic Portosystemic Shunt

*Data presented as mean, with range in parentheses.

\begin{tabular}{|c|c|}
\hline Etiology of Cirrhosis & N \\
\hline Hepatitis C & $15(27.7 \%)$ \\
\hline Alcoholic Liver Disease & $14(25.9 \%)$ \\
\hline NASH & $13(24.1 \%)$ \\
\hline Immune-mediated liver disease & $9(16.6 \%)$ \\
\hline Cryptogenic & $3(5.5 \%)$ \\
\hline Hepatocellular Carcinoma & $11(20.3 \%)$ \\
\hline
\end{tabular}

Table 2: Etiology of liver failure. 


\begin{tabular}{|c|c|c|}
\hline & Measured & Normal Values \\
\hline EXTEM MCF absolute [mm] & $53(11-70)$ & $50-72$ \\
\hline EXTEM ML mean [\%] & $5(7-17)$ & $<15$ \\
\hline EXTEM ML mean $>15 \%$ & $1 / 54(1.8 \%)$ & \\
\hline
\end{tabular}

Table 3: Fibrinolysis as measured by EXTEM maximum lysis (ML), defined as $M L>15 \%$

MCF: Maximum Clot Firmness; ML: Maximum Lysis

Measured data presented at mean, with range in parentheses.

\begin{tabular}{|c|c|c|c|}
\hline \multicolumn{4}{|c|}{ EXTEM ML $>$ APTEM ML } \\
\hline \multirow{3}{*}{ EXTEM ML $>15 \%$} & True & False & Total \\
\cline { 2 - 4 } & 1 & 0 & 1 \\
\cline { 2 - 4 } & 26 & 27 & 53 \\
\cline { 2 - 4 } & 27 & 27 & 54 \\
\hline
\end{tabular}

Table 4: Mc Nemar's table comparing both tests of hyperfibrinolysis.

McNemar's table describing results. One patient tested true for both definitions of hyperfibrinolysis. $\mathrm{ML}=$ maximum lysis

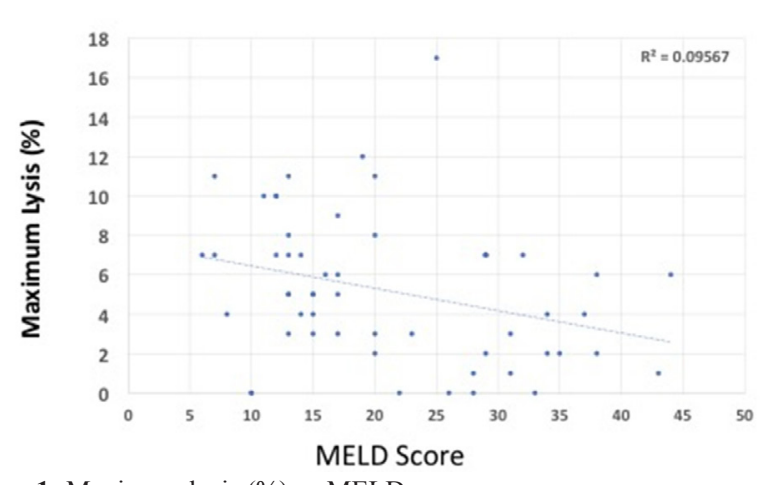

Figure 1: Maximum lysis (\%) vs MELD score.

$\mathrm{R}^{2}$ value showing Pearson coefficient.

\section{Discussion}

This is the first study, to our knowledge, of fibrinolysis in patients throughout their time on the liver transplant waiting list. Other studies have captured patients at the time of transplantation or the patients have not been listed $[3,10]$. Previous studies have shown that EXTEM maximum lysis correlates well with fibrinolysis. Spiel et al., validated EXTEM fibrinolysis using a lipopolysaccharide-induced model of fibrinolysis [4]. Levels of tissue plasminogen activity, plasminogen activator inhibitor, and anti-plasmin were used as markers of fibrinolytic activity. This has led to research in determining the best way to diagnose and treat hyperfibrinolysis, and authors have proposed transfusion algorithms based on early ROTEM parameters during liver transplant $[11,12]$. Recently, Abuelkasem et al., reported that FIBTEM is a more sensitive test for fibrinolysis than EXTEM [10]. This data was not available prior to our study. In that study, the authors reported a $23 \%$ incidence of hyperfibrinolysis at baseline, which is consistent with studies using the euglobulin lysis test as the confirmatory test. The authors used maximum lysis $>15 \%$ on
EXTEM or FIBTEM as well as correction of the lysis pattern on APTEM as confirmation of fibrinolysis. Their study enrolled 37 patients at the time of liver transplantation, whereas ours had a greater number of patients, but did not include FIBTEM tests. Depending on the definition of hyperfibrinolysis, we report an incidence between $1.8 \%$ and $50 \%$ in the pre-liver transplant population. Obviously, this wide range is not statistically significant and no definitive conclusions about the frequency and severity of hyperfibrinolysis can be drawn from the data. This would seem to support the notion that EXTEM, combined with APTEM, is a poor test alone for fibrinolysis. Platelet plugs contract during clot formation, the EXTEM tracing could be misinterpreted as fibrinolysis. In this case, the clot could still be strong, but the interpretation could lead to inappropriate antifibrinolytic therapy.

Because hyperfibrinolysis is still an ill-defined phenomenon in the liver transplant population, our data serves a useful purpose in helping anesthesiologists and surgeons in their efforts to initiate antifibrinolytics. ROTEM is relatively new in the United States and clinical experience is still emerging. The decision to start antifibrinolytics should not be based on laboratory data alone. In an otherwise dry surgical field, ROTEM evidence of hyperfibrinolysis should not be the only trigger for starting tranexamic or aminocaproic acid. Based on the results of our study, as well as similar research perfomed by other authors, we recommend using FIBTEM, EXTEM and APTEM channels, in addition to surgical bleeding conditions when making the decision to institute antifibrinolytic therapy.

Hyperfibrinolysis is a common, yet poorly defined pathology during cirrhosis. EXTEM tests such as ML $>15 \%$ and correction of the lysis pattern on APTEM give widely variable results when trying to identify patients with hyperfibrinolysis. Based on EXTEM and APTEM tests alone, no conclusions can be made about the incidence of hyperfibrinolysis in the pre-transplant population. On this data alone, we cannot recommend giving prophylactic antifibrinolytics to all patients when they arrive for liver transplantation.

\section{References}

1. Rana A, Petrowsky H, Hong JC, Agopian VG, Kaldas FM, et al. (2013) Blood transfusion requirement during liver transplantation is an important risk factor for mortality. J Am Coll Surg 216: 902-907.

2. Chandler WL (2016) Hyperfibrinolysis. In: Teruya J (ed.). Management of Bleeding Patients. Springer International Publishing AG, Cham, Switzerland. Pg no. 31-36.

3. Hu KQ, Yu AS, Tiyyagura L, Redeker AG, Reynolds TB (2001) Hyperfibrinolytic activity in hospitalized cirrhotic patients in a referral liver unit. Am J Gastroenterol 96: 1581-1586.

4. Spiel AO, Mayr FB, Firbas C, Quehenberger P, Jilma B (2006) Validation of rotation thrombelastography in a model of systemic activation of fibrinolysis and coagulation in humans. J Thromb Haemost 4: 411-416.

5. Levrat A, Gros A, Rugeri L, Inaba K, Floccard B, et al. (2008) Evaluation of rotation thrombelastography for the diagnosis of hyperfibrinolysis in trauma patients. Br J Anaesth 100: 792-797.

6. Rotem (2017) Thromboelastometry Methodology. Instrumentation Laboratory, Rotem, UK.

7. Dirkmann D, Görlinger K, Peters J (2014) Assessment of early thromboelastometric variables from extrinsically activated assays with and without aprotinin for rapid detection of fibrinolysis. Anesth Analg 119: 533-542. 
8. Gorlinger K, Iqbal J, Dirkman D, Tanaka KA (2016) Whole blood assay: Thromboelastometry. In: Teruya J (ed.). Management of Bleeding Patients. Springer International Publishing AG, Cham, Switzerland. Pg no. 207-356.

9. Nicolau-Raducu R, Ku TC, Ganier DR, Evans BM, Koveleskie J, et al. (2016) Epsilon-Aminocaproic Acid Has No Association With Thromboembolic Complications, Renal Failure, or Mortality After Liver Transplantation. J Cardiothorac Vasc Anesth 30: 917-923.

10. Abuelkasem E, Lu S, Tanaka K, Planinsic R, Sakai (2016) Comparison between thrombelastography and thromboelastometry in hyperfibrinolysis detection during adult liver transplantation. Br J Anaesth 116: 507-512.
11. Görlinger K, Tetsuro S, Dirkmann D, Planinsic RM, Saner FH. (2016) Bleeding Related to Liver Transplant. In: Teruya J (ed.). Management of Bleeding Patients. Springer International Publishing AG, Cham, Switzerland. Pg no. 263-280.

12. Solomon C, Schöchl H, Ranucci M, Schlimp CJ (2015) Can the Viscoelastic Parameter alpha-Angle Distinguish Fibrinogen from Platelet Deficiency and Guide Fibrinogen Supplementation? Anesth Analg 121: 289-301. 